\title{
Adding unmanned aerial vehicles to HIV supply chains in remote settings: modeling feasibility and cost in Turkana, Kenya
}

\author{
Caleb Parker ${ }^{1}$, Emily Evens ${ }^{2}$ (D) Kayla Stankevitz ${ }^{3}$, Margaret Eichleay ${ }^{4}$, Rick Homan ${ }^{4}$, Michael Loogos ${ }^{5}$, David Onyango ${ }^{6}$ \\ ${ }^{1}$ Behavioral, Epidemiological and Clinical Sciences, FHI 360. Durham, North Carolina, USA, 2 Health Services Research, FHI 360. Durham, North \\ Carolina, USA., ${ }^{3}$ HIV Programs, FHI 360. Durham, North Carolina, USA, ${ }^{4}$ Health Services Research, FHI 360. Durham, North Carolina, USA, 5 \\ AMPATHplus Program, Lodwar, Kenya, 6 Maetrics Consulting, Kisumu, Kenya \\ Keywords: kenya, hiv, supply chain, unmanned aerial vehicles \\ https://doi.org/10.29392/001c.28349
}

Journal of Global Health Reports

Vol. 5, 2021

\begin{abstract}
Background
Viral load (VL) testing of patients on antiretroviral therapy (ART) is critical to suppressing HIV. Timely transport of VL samples from health facilities to the laboratory for testing is often problematic in Turkana County, Kenya, and transportation barriers cause delays that can negatively impact the health of ART patients. Unmanned Aerial Vehicles (UAVs), or drones, are being promoted as a potential, novel way to reduce transportation times for laboratory samples and medical commodities. Our study models the use of UAVs in a remote area of Kenya to estimate costs and feasibility of their application for the Afya Nyota ya Bonde project, a large HIV service delivery program.
\end{abstract}

\section{Methods}

We gathered data from 12 Afya-supported facilities on VL sample transportation and turnaround time, ART drug transportation, and transportation practices and costs. The annual cost and average turnaround time for the transportation of VL samples and ART drugs was calculated using baseline data from the program to establish a comparison for scenarios that use a UAV. We then designed and costed four transportation scenarios that used UAVs to pick up VL samples and deliver ART drugs through a logistics company that will be adding UAV services.

\section{Results}

The modeled scenarios demonstrate that UAVs could transport nearly $100 \%$ of VL samples to the lab within the three-day goal compared to the $3 \%$ that are transported under the current system. Additionally, using UAVs could reduce the burden on health workers who must personally transport the samples, reducing opportunity costs resulting from facility closures due to provider absence. However, the UAV scenario cost more than three times the current costs (US\$ 56,350/Kenyan Shillings (KSh) 5,635,000 per year compared to between US\$172,878/Ksh 17,287,800 and US\$200,208/Ksh 20,020,800), and the consolidation of VL testing days required to accommodate the UAV may be problematic for ART patients.

\section{Conclusions}

As the cost of UAV transport declines, this technology could be an important tool for optimizing the transport of lab samples and medications.

In 2015, the Joint United Nations Programme on HIV and AIDS set the 95-95-95 targets with the goal of having 95\% of people living with HIV (PLHIV) tested, 95\% of those testing positive initiated into care and treatment, and $95 \%$ of those on treatment virally suppressed by the year $2030 .{ }^{1} \mathrm{Vi}$ ral suppression for PLHIV is essential to maintaining their health and, when the virus is suppressed to undetectable levels, HIV is not transmittable to others. ${ }^{2}$ Viral suppression is achieved through adherence to effective anti-retroviral drugs (ARVs) and is most effectively monitored through viral load (VL) testing. ${ }^{3,4}$ Achieving widespread VL testing coverage is critical to reaching the third 95\% goal; yet, hurdles in scale-up of VL testing persist in remote regions ${ }^{3-6}$ where transportation of VL samples is challenging and turnaround time (TAT) for VL test results is high. ${ }^{5}$ One potential solution to decrease TAT is the use of unmanned aerial vehicles (UAVs), or drones, to transport VL samples and ARVs. ${ }^{7}$

Interest in UAVs for transport of laboratory specimens and medical commodities has grown in recent years, and 
UAVs have been used to routinely transport medical supplies in a few countries. ${ }^{7-11}$ However, limited evidence exists on the cost of using UAVs for routine transport of laboratory specimens which require landing at remote clinics to pick up samples (termed directional transport). Additional questions remain about the feasibility of UAV services that are purchased through a logistics company, as opposed to a country or program purchasing and operating the units themselves, and when the distances required for transport are farther than 100 kilometers.

Determining the cost to utilize UAVs for routine transport of specimens is difficult considering the multiple assumptions that must be accounted for, including the type of UAV and the associated operational costs. A nationwide study in Liberia ${ }^{12}$ determined that fully replacing the motorbike transport program with UAVs for lab samples would be more expensive to operate. Phillips et al. ${ }^{13}$ conducted a costing analysis that compared UAV-based systems to ground-based for the transport of laboratory samples from health facilities to labs in Malawi and found higher costs but lower travel times when using small, battery-powered UAVs for transport. Haidari et al. ${ }^{14}$ modelled the feasibility and cost of using UAVs to transport vaccines in Mozambique, finding that for distances under $75 \mathrm{~km}$, UAVs increased availability of vaccines and decreased logistic costs. Others have studied how small UAVs might improve lab specimen transport over short distances. ${ }^{15-17}$ While these research activities are informative, their findings are not transferable to settings which require UAVs to fly longer distances with heavier cargo, and when UAV services are purchased from a third-party logistics company. Furthermore, UAV technology is progressing rapidly, and the UAVs that will soon be ready for commercial use can transport larger quantities over greater distances making them potentially more useful for last-mile logistics in remote locations.

\section{SPECIMEN TRANSPORT IN TURKANA COUNTY, KENYA}

Turkana County is a large rural and remote area in northwest Kenya. The Afya Nyota ya Bonde project (Afya), a 5-year USAID-funded project implemented by FHI 360 (2018 - 2022), is improving the capacity of commodity management and strengthening the laboratory network services for VL testing in order to reach the 95-95-95 targets. The Afya project supports 18 health facilities in Turkana County, 15 of which are in rural areas beyond the main town of Lodwar, the largest urban area in the county. Effective service delivery for PLHIV in Turkana County is hindered by several factors affecting transportation: underdeveloped road networks and unpredictable flash floods ${ }^{18}$; insecurity and violence ${ }^{19}$; vast distances between health facilities that offer HIV care and treatment services and the central laboratory where samples are tested; and unreliable transportation options.

Currently in Turkana County, health workers are responsible for transporting VL samples from their health facility to Lodwar, where the average one-way trip takes 4.5 hours. A courier service then transports the samples to a national reference laboratory in Eldoret, about an 8-hour drive oneway. All facilities collect VL samples using dried blood spots (DBS), drops of blood dried on filter paper packaged in an envelope for transport. Similarly complex is the distribution of ARVs. The Kenya Medical Supplies Authority (KEMSA) currently transports ARVs from national warehouses to Lodwar; then healthcare workers from around the county must travel to Lodwar to pick them up monthly. The challenges of transportation in this county severely impact service delivery: ARVs are often delayed reaching health facilities, and VL samples are delayed reaching the laboratory; with only $3 \%$ of all samples currently meeting the goal of a TAT less than four days. These delays prevent patients from receiving timely, clinically-informed therapies. ${ }^{20} \mathrm{Ad}$ ditionally, when health facility staff are away transporting VL samples and retrieving ARVs, provision of health services can be interrupted as small facilities must close when their one health care worker leaves.

The National AIDS/STD Control Programme (NASCOP), operated by the Kenyan Ministry of Health, records three segments of the total turnaround time for a VL sample: (1) the time between the collection of the sample from the patient to when it is received at the laboratory; (2) the time between the receipt of the sample at the laboratory until it is processed; and (3) the time between processing and dispatching the result. For the Afya Project, the ideal turnaround time for the first segment, referred to as TAT-1 in this text, is under four days. Official NASCOP data was available for 11 of the 15 remote health facilities in Turkana between January and March, 2018, the period immediately prior to the start of Afya project, and shows the TAT- 1 for all 270 VL samples taken during this time averaged 13 days (range 1-30), with 97\% of VL samples arriving four or more days after the sample was drawn. We used data prior to the start of the project as it was a realistic TAT without project support that was financially and logistically not sustainable by the Ministry of Health.

\section{STUDY OBJECTIVES}

Given the current transportation challenges faced in Turkana County, we aim to estimate the costs and potential time savings to the health system by replacing or supplementing ground transport of VL samples and medical commodities with transport by UAV. We model several different transportation scenarios at Afya-supported health facilities in Turkana County. For each scenario, we assess the potential costs and reduction in TAT- 1 as compared to the current transportation system. Our results are intended to inform government officials and decision-makers considering UAVs for transportation of commodities, as well as UAV manufacturers and logistics companies interested in using UAVs for this sector.

\section{METHODS}

This modeling study employed secondary analyses of health facility data, and de-identified data on the turnaround time for VL specimens. The study was reviewed by FHI 360's Office of International Research Ethics and was deemed to be a non-human subjects research activity. Use of programmatic data was approved by the Afya project as well as the Government of Kenya.

In this section, we discuss the assumptions of current 
practices, the data inputs we used for modeling the transportation network and informing the assumptions, and the logistical needs for UAV that was used to model transport scenarios. We then describe the modeling process for costing the current transportation system and the UAV transportation scenarios.

\section{DATA SOURCES}

We combined data from a national health database, health facility logs, and open-source spatial data. De-identified VL laboratory sample data were provided by NASCOP to determine sample origin and TAT for the Afya-supported health facilities. Data on the number of trips taken by health care workers to transport samples for VL testing, early infant diagnosis (EID) of HIV infection, TB, and others to Lodwar were collected by Afya staff from facility transportation logbooks. Facility locations in latitude and longitude were provided by the Afya project and were validated and corrected using satellite imagery from Google maps. The road network was downloaded from OpenStreetMap ${ }^{\circledR}{ }^{21}$ A UAV flight path network was created in ArcGIS, ${ }^{22}$ a geographic information system (GIS), by creating straight-line routes between all facilities. Average costs for ground-based transportation between health facilities and Lodwar town (including the staff per diem and the private transportation fare), and costs for the courier service from Lodwar to the laboratory were provided by the Afya project based on the local transport rates within Turkana. Estimated costs for UAV-based transportation and UAV specifications were provided by a logistics company in Kenya that is planning to add UAVs to its fleet.

\section{ASSUMPTIONS}

Transport scenarios were developed and modeled to compare cost and TAT for different transport method configurations. The scenarios are based on the following assumptions regarding the current methods of transporting VL samples and ARVs, and UAV specifications (Table 1). For this modeling exercise, we used an average setting for the system; the parameters are explained in this section, and the resulting averages for each variable are presented as results in Table 2 .

Currently, VL tests are not consolidated into weekly or monthly designated VL testing days. Instead, health workers provide VL testing services to antiretroviral (ART) patients as they are seen at a facility. This requires providers to either hold the VL samples until they accumulate enough to warrant transport to Lodwar, thus delaying TAT-1, or to travel to Lodwar after each day when tests were drawn from the patient, increasing the travel frequency, transportation costs, and provider time away from the facility. For UAV transport to be cost effective, however, we modeled consolidated VL testing days to reduce the number of flights. A UAV would be scheduled to pick up the VL samples the day after the ART patient is seen, which will also help reduce TAT-1. As the algorithm in the national guidelines ${ }^{23}$ provides the ART client with a 60-day window around the due date for their next test, each facility should offer at least one VL testing day per month. This will ensure each ART patient has at least two opportunities to access VL testing within their window.

In order to extrapolate data from logs and the NASCOP database on VL samples, which were used to model our scenarios, we generated three key variables.

- Expected number of VL samples per month, by facility: According to Kenya's national HIV testing algorithm, new clients require one VL test for every six months in their first year. Virally suppressed clients require one VL test per every 12 months; and non-suppressed clients require testing once every three months until they are virally suppressed. We assumed non-suppressed clients require two tests in 12 months. We computed the proportion of each client type from the study facilities in the NASCOP data and estimated the average number of VL tests per month per client.

- Expected amount of ART and other pharmaceuticals needed per month, by facility: For this activity, we based the quantity on cotrimoxazole-an antibiotic commonly prescribed for PLHIV-and the most commonly prescribed ART (TDF-3TC-EFV). About half of patients also need TB prevention drugs (INH and Pyridoxine). We calculated the total amount of these pharmaceuticals needed per ART client per month in kilograms based on every client requiring a onemonth supply of CTX and TDF-3TC-EFV, and half of clients requiring a one-month supply of TB drugs.

- Minimum number of VL testing days required by each facility every month: Collapsing the number of days when VL tests are offered for any one facility will reduce the number of trips a UAV must make to each facility. Using the NASCOP data, we identified the maximum number of tests per day conducted by each facility between January and March 2018 and consulted with the Afya staff to determine the appropriate maximum number of clients the average facility could offer VL testing services for in one day.

\section{MODELING THE CURRENT TRANSPORTATION SYSTEM}

We extrapolated the number of trips taken per facility to deliver VL samples to Lodwar from facility logs and then to Eldoret and pick up ARVs during the January to March 2018 quarter to provide a basis for estimating the annual groundbased transportation costs. This time frame was chosen to reflect the practices, costs, and targets achieved without the externally funded Afya program, which, through interventions to fund the transportation of VL samples, is reducing costs and improving service delivery. These annual costs for transportation of VL samples and ARVs were compared to the annual costs derived from modeling the UAV-based scenarios.

\section{DESIGNING THE UAV SCENARIOS}

Based on these assumptions, four scenarios (Table 3) were created that partially or fully replace the ground-based transportation network with a single UAV. All scenarios prioritize the transportation of VL samples, and some include UAV transportation of ARVs.

The scenarios differ by changing the settings of three pa- 
Table 1. Assumptions for scenario-building

\begin{tabular}{|c|c|}
\hline Input & Assumption \\
\hline \multirow[t]{2}{*}{$\begin{array}{l}\text { Facility } \\
\text { operations }\end{array}$} & $\begin{array}{l}\text { Each facility offers VL testing services as needed each month for ART clients. The expected number of VL } \\
\text { samples to collect each month is based on the total number of patients on anti-retroviral therapy (ART). }\end{array}$ \\
\hline & Each facility has an expected quantity of ARVs required each month based on the total number of ART patients. \\
\hline $\begin{array}{l}\text { ARV Origin } \\
\text { and } \\
\text { Destination }\end{array}$ & All ARVs are delivered by KEMSA to Lodwar town to be transported to health facilities by facility staff. \\
\hline $\begin{array}{l}\text { VL Sample } \\
\text { Origin and } \\
\text { Destination }\end{array}$ & $\begin{array}{l}\text { All viral load samples originate at the facility in which they were collected and are transported to a laboratory in } \\
\text { Eldoret. }\end{array}$ \\
\hline \multirow[t]{3}{*}{$\begin{array}{l}\text { Ground-based } \\
\text { transportation }\end{array}$} & $\begin{array}{l}\text { Healthcare workers travel to Lodwar to drop off VL samples and pick up ARVs, incurring costs of the fare for } \\
\text { transportation, additional costs for carrying boxes of ARVs back on local transport, and per diem for their time } \\
\text { away from their post. }\end{array}$ \\
\hline & VL samples are transported from Lodwar to Eldoret using a courier service with an estimated per-package fee. \\
\hline & $\begin{array}{l}\text { Travel times and transportation fare costs for health workers are incurred at a known average for each facility by } \\
\text { the Afya project. }\end{array}$ \\
\hline \multirow{4}{*}{$\begin{array}{l}\text { UAV } \\
\text { Specifications }\end{array}$} & The UAV has a maximum flight time/distance per tank of fuel and payload capacity in weight and volume. \\
\hline & $\begin{array}{l}\text { The UAV travels at an average speed along a straight-line, uninterrupted path between each origin and } \\
\text { destination, and can land/take-off from remote locations to perform reverse logistics. }\end{array}$ \\
\hline & The UAV is based in Lodwar. \\
\hline & The logistics company charges clients only by hours utilized per month for their transport services. \\
\hline
\end{tabular}

rameters:

- The number of health facilities where the UAV lands to pick up VL samples and drop off ARVs

- The UAV partially or fully supporting the pick-up of VL samples to transport to Lodwar

- The UAV partially, fully, or not at all supporting delivery of ARVs to health facilities

These parameters were chosen as they could impact the total UAV operational time, and thus the UAV costs. Exclusively transporting VL samples, picking up VL samples from centralized hub facilities instead of each facility, and delivering limited supplies of ARVs could all impact cost and TAT-1. All UAV models include the transport of VL samples from Lodwar to Eldoret by UAV.

\section{MODELING THE UAV SCENARIOS}

We produced a one-month schedule that reflects the average needs for VL sample transport and ARV delivery per facility for each scenario. For each week, the schedule begins with the UAV delivering ARVs and picking up VL samples on Tuesday morning from facilities that provided VL testing services on Monday. The samples are warehoused at Lodwar each day until they are flown to the lab in Eldoret. This allows facilities to provide VL testing starting Monday, then Tuesday through Thursday for pick up by UAV, and Friday (or earlier) as the day this UAV transports all VL samples to Eldoret. Additionally, this scheduling should allow the TAT- 1 to stay within the target. We temporally grouped UAV trips to facilities in the same area so that the UAV could make multiple stops in one trip, when distances allowed. We calculated the total weight in kilograms of deliveries and pickups per week for each facility.
Next, the weekly trip calculations were loaded into the Vehicle Routing Problem (VRP) solver in ArcGIS, which recognized the quantities of materials to transport from one location to another. Using the UAV flight path network, the tool was configured such that the UAV would travel from the base at Lodwar with the maximum payload weight to as many health facilities in one trip as possible and return to Lodwar once one or more of the constraints were met: maximum UAV distance on one tank of fuel, maximum operating time, or end of operational hours at the facilities. The UAV is modeled to land at its first health facility, have the ARV payload removed and have that facility's VL samples loaded within the maximum payload weight constraint. Depending on the scenario, the UAV may fly to more health facilities to pick up VL samples, returning to Lodwar once the weight constraint was met. If enough time remained in the same day to take additional trips, the UAV would refuel as needed and conduct another trip. The VRP solver would repeat this process until all trips were accomplished for that week.

The product of the VRP solver is a database of "sequences," providing the routes, travel times, payload delivered, and payload picked up for every trip. Figure 1, an illustrative example, shows these sequences, and how the resulting product could create a trip with multiple stops (sequences 1,2 , and 3), or a trip to only one facility and back to Lodwar (sequences 4 and 5). A solution was generated for every week for the generic one-month period. We summarized these flight sequences to calculate the total operational time (i.e., time spent preparing the UAV for flight, loading and unloading at the UAV base, refueling, flight time, and time spent loading and unloading at health facilities) per month for each scenario. This total time was used to calculate UAV costs annually. To these costs, we added 
Table 2. Calculated values by variable

\begin{tabular}{|c|c|}
\hline Facility Operations and Capacities & Value \\
\hline Expected number of VL samples per month & 0.1 per ART client \\
\hline Expected weight of ARVs per month & $0.2 \mathrm{~kg}$ per ART client \\
\hline Expected number of $\mathrm{VL}$ testing days per month & 1 day per $10 \mathrm{VL}$ tests \\
\hline \multicolumn{2}{|l|}{ Ground-Based Transportation Costs (USD) } \\
\hline Roundtrip transportation fare from health facilities to Lodwar & $\begin{array}{r}\text { Average: US\$24.39/Ksh2439 } \\
\text { Range: US\$12/Ksh1200-US\$35/ } \\
\text { Ksh3500 }\end{array}$ \\
\hline $\begin{array}{l}\text { Estimated fee for transporting one box of ARVs by ground-based transport from a health } \\
\text { facility to Lodwar (one box weighs approximately } 12 \mathrm{~kg} \text {, enough to supply } 60 \text { ART clients per } \\
\text { month) }\end{array}$ & US\$3/Ksh300 \\
\hline Average per diem provided to staff who are gone for more than half a day from their post & US $\$ 66.50 /$ Ksh66,500 \\
\hline Ground-based courier costs from Lodwar to Eldoret laboratory & US\$10/Ksh1000 per package \\
\hline \multicolumn{2}{|l|}{ Hourly UAV Usage Costs (USD) } \\
\hline 50-75 hours per month & US\$175/Ksh17,500 per hour \\
\hline 76-100 hours per month & US\$150/Ksh15,000 per hour \\
\hline 101-125 hours per month & US\$115/Ksh11,500 per hour \\
\hline $126+$ hours per month & US\$100/Ksh10,000 per hour \\
\hline \multicolumn{2}{|l|}{ UAV Specifications and Operations } \\
\hline Total number of UAVs available & 1 \\
\hline Base location of UAV & Lodwar \\
\hline UAV operation start time & $7 \mathrm{am}$ \\
\hline UAV operation end time & $7 \mathrm{pm}$ \\
\hline Required service time for prep work and loading ARVs & 30 minutes \\
\hline Required service time for unloading VL samples & 30 minutes \\
\hline Required service time for refueling the UAV & 30 minutes \\
\hline Maximum kilograms the UAV can transport & $10 \mathrm{~kg}$ \\
\hline Total flight time per tank of fuel & 600 minutes \\
\hline Total flight distance per tank of fuel & $300 \mathrm{~km}$ \\
\hline Average UAV speed & $75 \mathrm{kph}$ \\
\hline \multicolumn{2}{|l|}{ UAV Landing Site Operations } \\
\hline Time needed to load/unload supplies and conduct checklist & 30 minutes \\
\hline Earliest time the UAV can arrive at a facility & $7 \mathrm{am}$ \\
\hline Latest time the UAV can depart from facility & $5 \mathrm{pm}$ \\
\hline
\end{tabular}

any additional ground-based transportation costs using the known fare, box surcharge, and per diem rates earned by facility staff when away from facility.

\section{RESULTS}

Data on the number of trips undertaken to transport VL samples between January and March 2018 was available for 11 of the 15 facilities. For the four facilities where no information was collected, only one was found in the NASCOP database to show it had sent samples. Therefore, 12 of the 15 facilities conducted VL testing in this quarter. According to the health facility transportation logbook records from
January to March 2018, health care workers took 63 total trips from 12 facilities, carrying between 1 and 35 VL samples per trip. (Data discrepancies exist between the NASCOP data and the health care logbooks for most facilities.) The latest available data from NASCOP (September 2017) showed 1,316 ART clients in care at these facilities (average $=87.7$ clients per facility; range $=3$ to 231 ).

The calculated values for the variables included in the models are listed in Table 2. Based on these data and conversations with the Afya staff, we estimated that one ART client would require $0.1 \mathrm{VL}$ samples per month and 0.2 kilograms of ARVs per month, and that the average facility would provide a minimum of one VL testing day per month for up to 10 ART clients. To generate monthly totals for VL 
Table 3. UAV-Modified Scenarios by landing location, and degree of support for VL pickup and ARV delivery

\begin{tabular}{|c|c|c|c|c|}
\hline Scenario & $\begin{array}{l}\text { At which health } \\
\text { facilities does the } \\
\text { UAV land?* }\end{array}$ & $\begin{array}{l}\text { Does the UAV partially, or } \\
\text { fully support the pick-up of } \\
\text { VL samples and transport to } \\
\text { Lodwar? }\end{array}$ & $\begin{array}{l}\text { Does the UAV partially, } \\
\text { fully, or not at all support } \\
\text { transport of ARVs from } \\
\text { Lodwar to facilities? }\end{array}$ & $\begin{array}{l}\text { Does the UAV } \\
\text { transport VL } \\
\text { samples from } \\
\text { Lodwar to } \\
\text { Eldoret? }\end{array}$ \\
\hline $\begin{array}{l}\text { Scenario 1: } \\
\text { Dedicated } \\
\text { VL }\end{array}$ & All sites & $\begin{array}{l}\text { Picks up all VL samples from } \\
\text { all sites }\end{array}$ & Transports no ARVs & Yes \\
\hline $\begin{array}{l}\text { Scenario 2: } \\
\text { VL plus } 1 \\
\text { ARV } \\
\text { shipment/ } \\
\text { month }\end{array}$ & All sites & $\begin{array}{l}\text { Picks up all VL samples from } \\
\text { all sites }\end{array}$ & $\begin{array}{l}\text { Transports at least one ARV } \\
\text { package per month? per } \\
\text { facility }\end{array}$ & Yes \\
\hline $\begin{array}{l}\text { Scenario 3: } \\
\text { VL plus } \\
\text { select ARV } \\
\text { shipment/ } \\
\text { month }\end{array}$ & $\begin{array}{l}\text { Selected facilities } \\
\text { (Hubs) (quantity to } \\
\text { be determined } \\
\text { during modeling) }\end{array}$ & $\begin{array}{l}\text { Picks up all VL samples from } \\
\text { selected facilities; remaining } \\
\text { sites must use ground to } \\
\text { reach nearest hub }\end{array}$ & $\begin{array}{l}\text { Transports at least one ARV } \\
\text { package per selected facility }\end{array}$ & Yes \\
\hline $\begin{array}{l}\text { Scenario 4: } \\
\text { VL plus } \\
\text { ARV }\end{array}$ & All sites & $\begin{array}{l}\text { Picks up all VL samples from } \\
\text { all sites }\end{array}$ & $\begin{array}{l}\text { Transports all ARVs to all } \\
\text { facilities }\end{array}$ & Yes \\
\hline
\end{tabular}

*Only 13 are landing sites because in two instances, two facilities are very close to each other and can easily be reached by health workers from both sites.

samples and ARVs by facility, these values were multiplied by the number of ART patients at each facility. All costs are listed in US dollars (USD), and costs originally provided in Kenyan shillings (KSH) were converted to USD where 100 $\mathrm{KSH}=1$ USD.

Based on these data and optimal routing, we modeled the four UAV-modified scenarios and estimated TAT-1 and cost, which are compared to the current configuration model (Table 4).

\section{RESULTS OF THE CURRENT SYSTEM}

We estimated the annual cost to transport all VL samples from health facilities to the laboratory in Eldoret, and to transport ARVs from Lodwar to each health facility to total US $\$ 56,350 / K s h 5,635,000$. Only $3 \%$ of the VL samples met the TAT-1 goal of under four days. To provide an annual cost, we assume the same number of VL sample trips and trips to pick up ARVs that happened between January and March 2018 will happen for the rest of the year. Only the 12 facilities that recorded sending VL samples in their logbooks between January and March 2018 are included in this scenario. No data were collected on the trips health care workers took to retrieve ARVs from Lodwar and were assumed that one trip per facility was taken each month, incurring the average fares and surcharges for carrying boxes. Based on conversations with providers we estimated that each trip took providers away from their facilities from between one-and-a-half and two days.

\section{UAV-BASED SCENARIO RESULTS}

Scenario 1: In this scenario, UAVs completely replace ground-based transportation of VL samples by landing at all facilities at least once per month to pick up the samples and take them to Lodwar. No ARVs or other supplies are car-

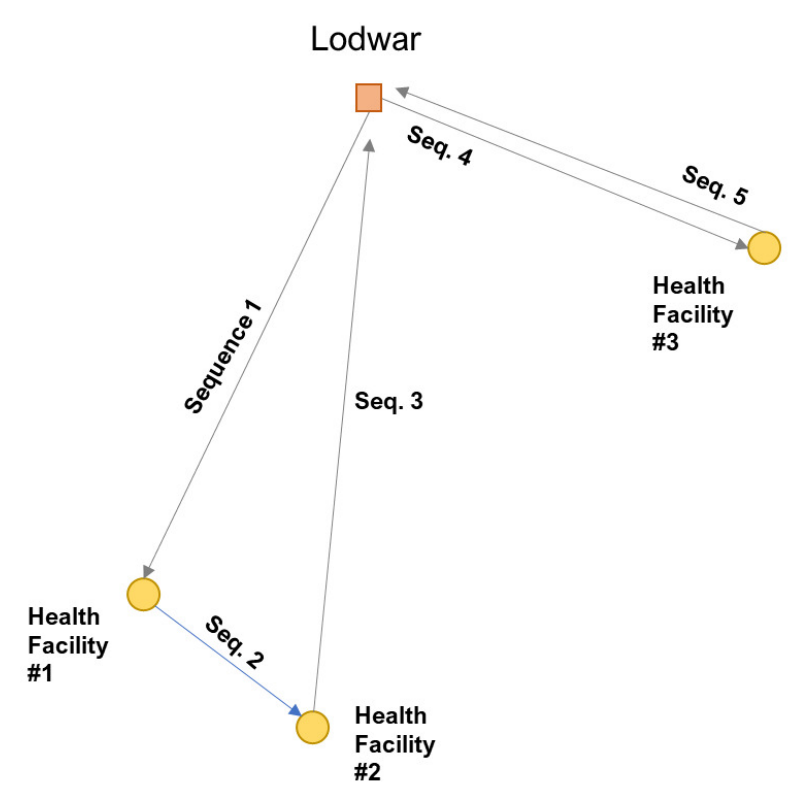

Figure 1. Example of UAV flight sequences from Lodwar to health facilities and back.

ried by UAVs, meaning health workers still need to travel to Lodwar to pick them up. The estimated annual cost for this model is US $\$ 172,878 / \mathrm{Ksh} 17,287,800$ which is 3.1 times the cost of the current system and is inclusive of the trips health care workers would have to take to pick up ARVs from Lodwar. While all VL samples would reach the lab in under four days, all health facilities would still see similar rates of staff being unavailable as they travel to Lodwar to pick up ARVs.

Scenario 2: This scenario builds on Scenario 1 by adding partial distribution of ARVs via UAV. Under this scenario, the UAV lands at every facility, delivering 10 kilograms of 
Table 4. Turnaround time and cost results by scenario

\begin{tabular}{|c|c|c|c|}
\hline Scenario & $\begin{array}{l}\text { TAT-1 }<4 \\
\text { days }\end{array}$ & $\begin{array}{l}\text { Total Annual Cost (US Dollars/Kenyan } \\
\text { Shillings) }\end{array}$ & $\begin{array}{l}\text { Cost increase factor from Current } \\
\text { system }\end{array}$ \\
\hline $\begin{array}{l}\text { Current } \\
\text { System }\end{array}$ & $3 \%$ & $\$ 56,350 / 5,636,000$ & \\
\hline Scenario 1 & $100 \%$ & $\$ 172,878 / 17,287,800$ & 3.1 \\
\hline Scenario 2 & $100 \%$ & $\$ 183,363 / 18,336,300$ & 3.3 \\
\hline Scenario 3 & $100 \%$ & $\$ 179,401 / 17,940,100$ & 3.2 \\
\hline Scenario 4 & $94 \%$ & $\$ 200,208 / 20,020,800$ & 3.6 \\
\hline
\end{tabular}

ARVs to each facility for every trip taken to pick up VL samples (except in two instances where the UAV lands at one of two neighboring facilities; in that case only one of the facilities would receive the package per delivery). After delivery of ARVs, the UAV picks up VL samples and returns to Lodwar. For Scenario 2, the estimated cost is US\$183,363/ Ksh18,336,300 or 3.3 times the current costs. All VL samples would reach Eldoret in under four days, but health care workers from all but two facilities would still be required to travel once a month to Lodwar to pick up ARVs that were not transported by the UAV because it could not carry all required ARVs.

Scenario 3: In this scenario, the UAV lands only at four "hub" facilities and three other facilities that are too remote to travel to a hub or to Lodwar easily. Facilities where the UAV does not land are required to travel to their closest hub via ground-based transport. Facilities that were within 20 $\mathrm{km}$ from other facilities were considered part of a hub system and the facility with the most ART clients in each system was chosen as the hub. In one instance, a facility was within $10 \mathrm{~km}$ of a hub, but the road network used to reach the hub was significantly challenging such that it was considered a remote site. Only hub or remote sites would receive one $10 \mathrm{~kg}$ package of ARVs per VL trip, but all sites would have their VL samples ultimately transported to Lodwar from the hub using the UAV. Two facilities were able to receive all ARVs this way, but the other facilities still required health workers to travel once per month to Lodwar to get their month's supply of ARVs.

Reducing the number of landing sites could reduce costs, however as the UAV lands at four hubs and three remote sites in this scenario, the costs are similar to previous scenarios. Eight sites would still have to use ground transportation to reach their UAV hub, and all sites would still require staff to travel to Lodwar to pick up all their ARVs. The cost, including additional ground-based transport is US $\$ 179,401 /$ Ksh17,940,100 or 3.2 times the current costs.

Scenario 4: The UAV completely replaces the groundbased distribution network of ARVs from Lodwar to each facility and includes the transportation of all VL tests from each site to Lodwar. The number of flights per month is based on the amount of ARVs required as more flights are needed to transport ARVs than are to pick up VL tests. Seven facilities require only one UAV trip per month to pick up all VL samples and deliver all needed ARVs, while eight facilities will be visited by the UAV an additional one to two times per month compared to what is needed to pick up VL samples only. Health care workers would not need to travel for ARVs, increasing their availability at the clinic. This cost, however, is the highest, at US\$200,208/ Ksh20,020,800 or 3.6 times the current amount.

Because of the increased demand on a single UAV, during two of the four weeks the UAV would have to fly to Eldoret on the following Saturday, which means the batch of VL samples it transported from the facilities visited on Tuesday and housed at Lodwar would not reach Eldoret for five days, two days beyond the TAT-1 goal.

\section{DISCUSSION}

In our modeled scenarios, using a UAV reduces the first segment of turnaround time of VL samples while increasing coverage to the $95 \%$ target achieving key goals in this region. Additionally, when providers are not responsible for the transport of samples, use of UAVs ensures that health care providers are not taken away from their duties to transport samples for one to two days a month. The opportunity costs of using health care providers for transporting samples are not included in these models but are estimated to be considerable especially given shortages of trained health workers are common in Kenya and Turkana in particular.

However, using UAVs to transport VL samples in Turkana County would be significantly more expensive than the current ground-based transportation system. The least expensive UAV scenario is still more than three times the cost of the current system and the current costs of ground-based transportation are already high in Turkana. When compared to facilities in the more densely populated Siaya County, Kenya, (the only county with available data on transportation costs for VL samples in Kenya) the ground-based transportation cost for 4,377 VL samples (DBS and whole blood) totaled US\$53,294/Ksh5,329,400 in 2014-approximately double the number of samples for about the same transport cost. ${ }^{24}$ However, the price of the UAV and the subsequent hourly rate charged by the logistics company operating the UAV will likely decrease as manufacturers' costs decrease over time, a common trend with any emerging technology. Additionally, expanding the use of UAVs to cover transport of more items than included in the modeling in this paper may also help reduce costs.

While UAVs are currently cost-prohibitive under this model, they could become more cost-competitive over time 
if the technology proves successful and costs are reduced. Other aspects of systems-level feasibility should also be considered. For the UAV configurations to work as modeled, each facility must consolidate their VL testing schedule to aggregate client visits for VL testing to specific days so that the UAV can land at multiple sites on the same day. This would likely require central coordination, which may incur more costs. As a scrub desert with low population density and limited infrastructure such as power lines or tall buildings, Turkana is an excellent location for a UAV program. While weather related issues must be considered before the development of a UAV program, these did not present a critical problem for the type of UAV used for modeling. Additionally, a UAV would likely not be affected by the same factors that currently limit ground-based transport (e.g., community violence, road bandits, roads closed from flooding) and may provide an alternative that offers continual, routine transportation solutions.

While reducing the number of days when VL tests are collected improves efficiency when using UAVs and decreases TAT, this practice could complicate patient care as clients in large and rural areas currently struggle with travel to facilities. If transportation is a challenge, then it is also probably difficult for patients to reach facilities. Unless facilities are able to see all patients on these VL testing days, that means asking patients to possibly make multiple trips, thus transferring the burden from the health system to the patient. Also, in some places, health systems are moving away from ART clinic days, and are instead integrating HIV treatment into general outpatient services to reduce stigma. In light of this, implementing specific VL testing days just to accommodate UAV transport, may be a problematic approach.

We recognize this modeling activity has several limitations. Our scenarios are focused on transporting VL samples and to varying degrees ARVs, but EID and TB tests are also important laboratory samples that require transportation. EID tests are required to be taken to a laboratory within a day of the birth of a child and returned to the health center as soon as possible and were not included in our model for this reason. To keep this timeframe even when a UAV is fully utilized for VL sample transport, staff may still have to use ground-based transportation. However, EID samples could easily fit on the UAV if the timing was appropriate. TB sputum specimens require more space, more rapid transport and must be kept at a constant cold temperature, complicating transport by UAV unless those UAVs are designed specifically to do so.

Finally, our estimated UAV costs come from one company which may not reflect charged by other providers. However, while other logistics companies may have similar plans to add UAVs to their fleets, we believed this company's understanding of the UAV landscape and local regulations, as well as their involvement in Turkana made them an ideal source. We also relied on this company to provide the estimated specifications of an aircraft they are considering purchasing once on the market and to vet the UAV manufacturer. The logistics company indicated the UAV model used in this activity was the best fit for the distances and payload required. Our modeling provides a first glimpse on the cost and design of a UAV-augmented laboratory transport sys- tem. While our results provide an estimate of the costs of UAV assisted lab transport given the best data available at the time, we also acknowledge rapid changes to UAV technology will impact the cost and time of transport. Finally, once the technology is available, additional costs including operation and maintenance, program oversight, and training must also be considered.

\section{CONCLUSIONS}

Increasing the number of ART clients who are virally suppressed to reach the 95-95-95 goals requires establishing and maintaining connections between health facilities in remote areas and laboratories and commodity storehouses. Introduction of UAVs into the HIV supply chain for facilities in this study could reduce the turnaround time for VL samples and support positive health outcomes for the ART clients. However, using current, Turkana-specific cost estimates, UAVs are not cost competitive with the current ground-based transportation system as we have modeled in these scenarios, and the practice of consolidating VL testing days to accommodate UAVs may not be practical for patient care. As costs decrease and technology improves to allow for increases in carrying capacity and the distance that it can travel before refueling, as well as more frequent flights, a UAV-modified transportation system for VL samples and ARVs may one day be logistically and financially feasible for Turkana.

\section{ACKNOWLEDGMENTS}

The authors would like to thank the Turkana County, Kenya Health Department for their important support of this work.

\section{FUNDING}

This research did not receive any specific grant from funding agencies in the public, commercial, or not-for-profit sectors.

\section{AUTHORSHIP CONTRIBUTIONS:}

CP (CParker@fhi360.org): Conceptualization; data curation; formal analysis; investigation; methodology; project administration; resources; software; visualization; writing original draft.

EE (EEvens@fhi360.org): Conceptualization, funding acquisition, investigation, methodology, writing original and draft, writing review and editing.

KS (KStankevitz@fhi360.org): Conceptualization, methodology, validation, writing review and editing.

ME (MEichleay@fhi360.org): Conceptualization, funding acquisition, methodology, writing review and editing.

RH (RHoman@fhi360.org): Methodology, writing review and editing.

ML (MLoogos@gmail.com): Methodology, writing review and editing.

DO (으ungid@gmail.com): Investigation, writing review and editing. 


\section{COMPETING INTERESTS}

The authors completed the Unified Competing Interest form at www.icmje.org/disclosure-of-interest/ (available upon request from the corresponding author), and declare no conflicts of interest.

\section{CORRESPONDENCE TO:}

Emily Evens, $\mathrm{PhD}, \mathrm{MPH}$

FHI 360359 Blackwell Street, Suite 200 Durham, NC 27701, USA

Submitted: May 11, 2021 GMT, Accepted: August 20, 2021 GMT 


\section{REFERENCES}

1. World Health Organization. Joint United Nations Program on HIV/AIDS. HIV/AIDS Programme. Strengthening Health Services to Fight HIV/AIDS. Guidance on Provider-Initiated HIV Testing and Counselling in Health Facilities. WHO; 2007.

2. Eisinger RW, Dieffenbach CW, Fauci AS. HIV viral load and transmissibility of HIV infection: Undetectable equals untransmittable. JAMA. 2019;321(5):451-452. doi:10.1001/jama.2018.21167

3. Friedland GH, Williams A. Attaining higher goals in HIV treatment: The central importance of adherence. AIDS. Published online September 13, 1999:S61-72.

4. Wainberg MA, Friedland G. Public health implications of antiretroviral therapy and HIV drug resistance. JAMA. 1998;279(24):1977-1983. doi:10.10 01/jama.279.24.1977

5. Lecher S, Ellenberger D, Kim AA, et al. Scale-up of HIV viral load monitoring-seven sub-Saharan African countries. MMWR Morb Mortal Wkly Rep. 2015;64(46):1287-1290. doi:10.15585/mmwr.mm6446 a3

6. Roberts T, Cohn J, Bonner K, Hargreaves S. Scaleup of routine viral load testing in resource-poor settings: current and future implementation challenges. Clin Infect Dis. 2016;62(8):1043-1048. do i:10.1093/cid/ciw001

7. Krey M. Drones: Application and business models in Swiss hospitals. In: The Hawaii International Conference on System Science, Waikoloa Village, 2-6 January 2018. Hawaii International Conference on System Sciences; 2018:3081-3089. doi:10.24251/hics $\underline{\text { s.2018.389 }}$

8. Drones deliver blood and medical supplies in Rwanda. BBC. May 1, 2018.

9. Drone to transport laboratory samples across Lake Zurich. Swiss Post. June 22, 2018.

10. UAV for Payload Delivery Working Group (UPDWG). Medical Drone Delivery Database. Published online 2020. Accessed October 28, 2020. htt ps://www.updwg.org/md3/

11. Banker S. North Carolina Hospital Chain Beings Drone Deliveries of Medicines. Forbes Magazine. August 18, 2020.
12. Ochieng WO, Ye T, Scheel C, et al. Uncrewed aircraft systems versus motorcycles to deliver laboratory samples in west Africa: A comparative economic study. Lancet Glob Health. 2020;8(1):143-151. doi:10.1016/s2214-109x(19)3046 $\underline{4-4}$

13. Phillips N, Blauvelt C, Ziba M, et al. Costs Associated with the Use of Unmanned Aerial Vehicles for Transportation of Laboratory Samples in Malawi. VillageReach; 2016.

14. Haidari LA, Brown ST, Ferguson M, et al. The economic and operational value of using drones to transport vaccines. Vaccine. 2016;34(34):4062-4067. $\underline{\mathrm{d}}$ oi:10.1016/i.vaccine.2016.06.022

15. Berman JS. Drone-based delivery of clinical specimens in a rural environment: A feasibility study (Doctoral dissertation). Published online 2017.

16. Meier P, Soesilo D. Using drones for medical payload delivery in Papua New Guinea. Drones in Humanitarian Action: Case Study. 2015;(2).

17. Rabta B, Wankmüller C, Reiner G. A drone fleet model for last-mile distribution in disaster relief operations. Int J Disaster Risk Reduct. 2018;28:107-112. doi:10.1016/j.ijdrr.2018.02.020

18. Turkana County Investment Plan 2016-2020.

19. Johannes EM, Zulu LC, Kalipeni E. Oil discovery in Turkana County, Kenya: A source of conflict or development? Afr Geogr Rev. 2015;34(2):142-164. do $\mathrm{i}: 10.1080 / 19376812.2014 .884466$

20. Minior T, Douglas M, Edgil D, et al. The critical role of supply chains in preventing human immunodeficiency virus drug resistance in low-and middle-income settings. J Infect Dis. 2017;216(suppl_9):S812-815. doi:10.1093/infdis/jix40 3

\section{OpenStreetMap contributors.}

22. ESRI. ArcGIS Desktop: Release 10.5. Environmental Systems Research Institute; 2019.

23. Ministry of Health, National AIDS \& STI Control Programme. Guidelines on Use of Antiretroviral Drugs for Treating and Preventing HIV Infections in Kenya 2016. NASCOP; 2016. 
24. Cintron C, Mudhune V, Haider R, et al. Costs of HIV Viral Load and Early Infant Diagnosis Testing in Kenya. Health Finance and Governance Project, Abt Associates Inc; 2017. 